\title{
La modélisation micro- et nano-dosimétrique au CPAT
}

\author{
M. TERRISSOL ${ }^{1}$
}

(Manuscrit reçu le 6 novembre 2006, accepté le 28 février 2007)

\begin{abstract}
RÉSUMÉ Depuis une trentaine d'années, le Centre de Physique Atomique de Toulouse a développé des codes de calcul du transport des particules dans la matière et plus particulièrement ces dernières années il s'est orienté vers l'étude des risques biologiques des rayonnements. Ce papier décrit les différentes étapes physique, physico-chimique et chimique de la simulation jusqu'à environ une microseconde pour évaluer les distributions des dommages initiaux à l'origine des désordres radiobiologiques subséquents. Deux exemples de calcul des dommages à l'ADN sont exposés : 1) dommages causés par des rayons $X$ ultra mous et 2 ) complexité des dommages causés par l'émission Auger et la fluorescence dues à la réorganisation interne dans le cisplatine après photoactivation de la couche $K$ du platine.
\end{abstract}

ABSTRACT Micro- and nano-dosimetry modelling at CPAT.

Since about thirty years the Centre de Physique Atomique de Toulouse has developed particle transport codes in matter and more specially last years has stressed the biological radiation induced risk. This paper describes the various stages of the particle transport: the physical stage, the physico-chemical stage and the chemical one, during the very early passage of particles in matter say up to one microsecond. Two examples of DNA damage evaluation are given: 1) DNA damage caused by ultrasoft X-rays, and 2) damage complexity created by Auger and fluorescence emissions due to the photoactivation of platinum $\mathrm{K}$-shell in the cisplatinum drug.

Keywords: DNA structures / radiations transport / early damage modeling

\section{Introduction}

Depuis le début des années 1970, le CPAT développe des codes de calcul du transport des particules dans la matière et s'est tout naturellement orienté avec le concours de la Communauté européenne vers l'évaluation des risques de cancer pour l'homme dus à des faibles doses de rayonnement. L'ADN étant la cible privilégiée, nos codes sont bâtis pour évaluer les distributions inchoatives des dommages initiaux jusqu'à des temps de l'ordre de la microseconde après le passage des particules dans l'environnement chromosomique. Ces distributions seront ensuite utilisées par d'autres modélisateurs pour évaluer les risques radiobiologiques.

\footnotetext{
${ }^{1}$ CPAT, université Paul Sabatier, 118 route de Narbonne, 31062 Toulouse, France.
} 


\section{Interaction ravonnement-ADN}

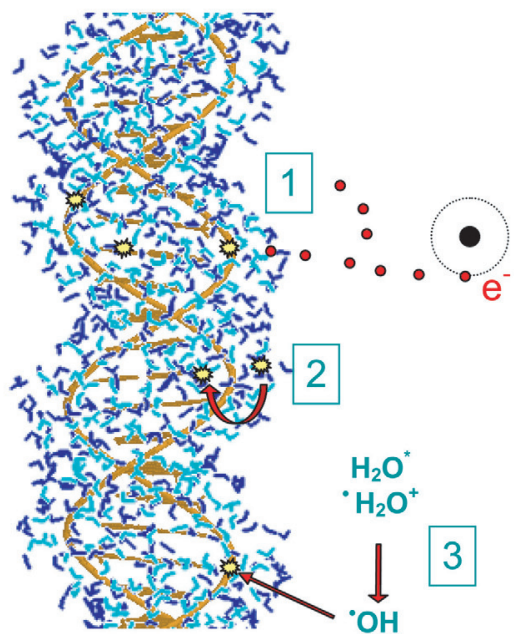

RAYONNEMENT

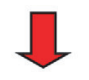

1. DIRECT

$\mathrm{t}<10^{-15} \mathrm{~s}$

\section{QUASI-DIRECT}

transferts de charges $t<10^{-12} \mathrm{~s}$

\section{INDIRECT}

$\mathrm{t}<10^{-8} \mathrm{~s}$

Figure 1 - Chronologie de l'absorption primaire du rayonnement.

Chronology of the primary absorption of radiation.

\section{Modélisation de l'effet des rayonnements sur la fibre chromosomique}

L'absorption de l'énergie d'un rayonnement ionisant par la matière vivante provoque un ensemble de transformations physiques et chimiques conduisant à un effet biologique final. Cette absorption primaire se déroule à des temps inférieurs à la femtoseconde, et à environ $10^{-9}-10^{-8}$ seconde on estime que toutes les espèces chimiques issues de la radiolyse de l'eau ont disparu, soit par neutralisation, soit par réaction avec les biomolécules. Ces effets primaires découlent toujours initialement d'une distribution plus ou moins structurée des espèces ionisées et excitées le long de la trajectoire de la particule incidente et des électrons secondaires créés. La description des événements subséquents est extrêmement complexe, régie par des processus physiques, chimiques et biologiques.

Il est d'usage de diviser la séquence des événements reliant le passage de la particule à l'apparition des effets biologiques selon une échelle de temps (Fig. 1) :

- à $\mathrm{t}<10^{-15} \mathrm{~s}$, on parle d'étape physique qui correspond à l'absorption de l'énergie du rayonnement ; 
- à $10^{-15} \mathrm{~s}<\mathrm{t}<10^{-12} \mathrm{~s}$, c'est l'étape physico-chimique qui est une phase de relaxation et de distribution de l'énergie absorbée ;

- à $10^{-12} \mathrm{~s}<\mathrm{t}<10^{-3} \mathrm{~s}$, c'est l'étape chimique, durant laquelle les espèces créées diffusent, réagissent, altèrent le milieu ;

- de quelques secondes à plusieurs années, l'étape biologique pendant laquelle les mécanismes complexes de la machine biologique mènent aux effets macroscopiques observés.

Le code développé au CPAT se propose donc d'évaluer les dommages primaires $10^{-6}$ seconde après le passage des particules en s'appuyant sur les phénomènes physiques qui décrivent le passage du rayonnement et sur le développement de modèles cibles suffisamment réalistes. Ce code possède les caractéristiques suivantes :

- calcule des cibles d'ADN simples et d'ordre supérieur : plasmide, nucléosome, fibre chromosomique en milieu homogène ou hétérogène ;

- transporte des photons et des électrons par méthode de Monte Carlo, pour des énergies inférieures à $200 \mathrm{keV}$;

- inclut les réorganisations radiatives (fluorescence) et non radiatives (électrons Auger) ;

- inclut les transferts de charges le long des biomolécules ;

- inclut la réparation chimique primaire ;

- évalue les dommages primaires à $10^{-6}$ seconde.

\subsection{Calcul des données de base}

Pour modéliser le transport, il faut avoir une représentation $\mathrm{x}, \mathrm{y}, \mathrm{z}$ des structures d'ADN simples et d'ordre supérieur (Fig. 2).

Les données de base pour le calcul de l'étape physique sont les diverses structures de l'ADN et toutes les sections efficaces totales et différentielles régissant les différentes interactions. Ces sections efficaces étant vraiment le fondement de la méthode développée, la recherche de ces données et/ou leur calcul nécessite un grand investissement et une attention permanente. À titre d'exemples, sont représentées sur la figure 3 les sections efficaces d'ionisation de la cytosine calculées par la méthode Binary-Encounter-Bethe, à l'aide des paramètres orbitaux évalués par le logiciel GAMESS, et sur la figure 4, les sections efficaces totales d'ionisation par impact électronique de tous les constituants de l'ADN.

La prise en compte de la réorganisation atomique ou moléculaire lors d'une ionisation en couche interne nécessite la connaissance de tous les niveaux et populations des atomes et molécules mis en jeu afin d'obtenir les probabilités de transition et les distributions d'électrons Auger et de rayons $\mathrm{X}$ de fluorescence émis. 
M. TERRISSOL

ADN linéaire hydraté
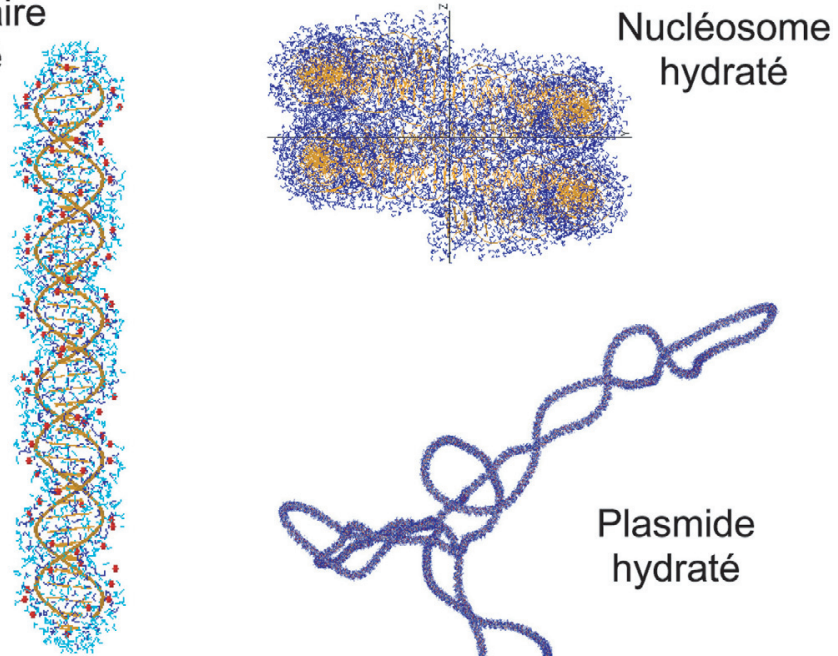

hydraté
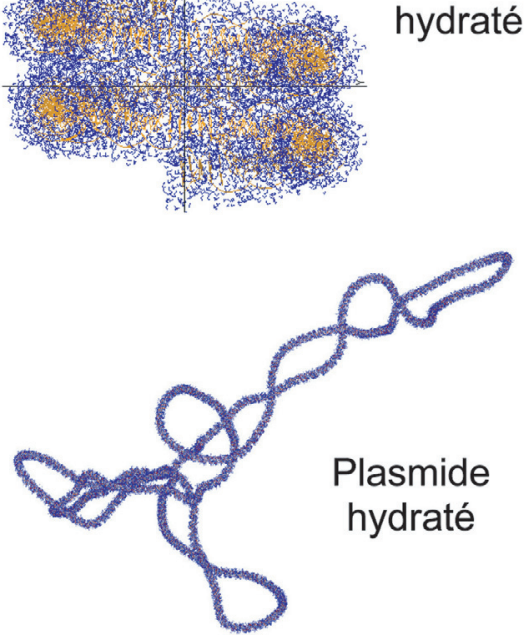

Figure 2 - Différentes structures d'ADN modélisées. Nucléosome hydraté de 146 paires de bases plus 806 protéines des histones soit environ 30000 atomes. Plasmide pBr322 hydraté de 5372 paires de bases soit environ 500000 atomes.

Some modelled DNA structures. Core particle of 146 base pairs with 806 histon proteins i.e. about 30,000 atoms. Hydrated pBr322 Plasmid with 5,372 base pairs i.e. about 500,000 atoms.

Paramètres $\mathrm{B}$ et $\mathrm{N}$ calculés avec

GAMESS, $U$ approximé connaissant

la population de chaque $O M$
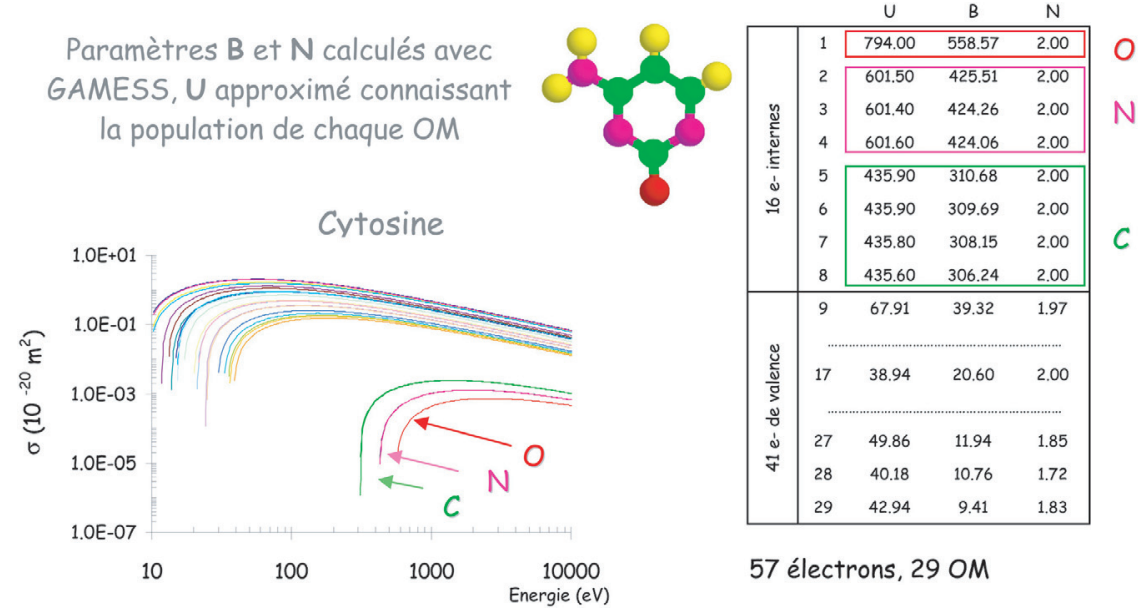

57 électrons, 29 OM

Figure 3 - Sections efficaces d'ionisation par les électrons de la cytosine.

Ionisation cross sections for electrons on cytosine. 


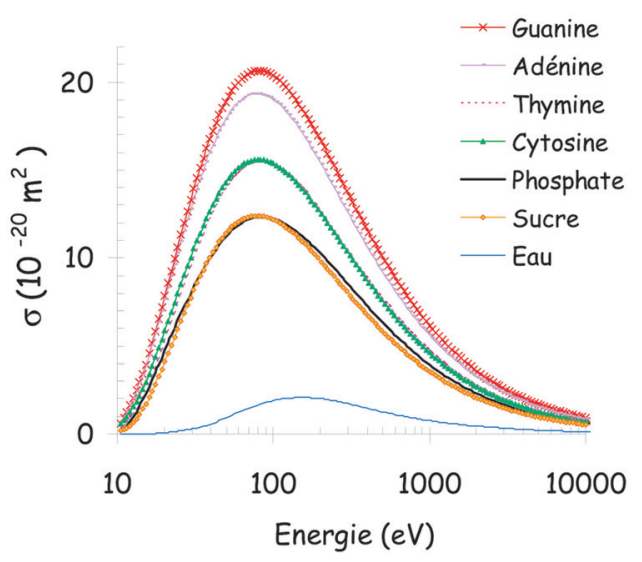

Figure 4 -Sections efficaces totales d'ionisation des constituants de l'ADN.

DNA total ionisation cross sections.

Pour l'étape physico-chimique, il faut déterminer tous les taux et schémas de transferts phosphate-base, sucre-base, base-base, couche d'hydratation-ADN et la radiolyse de l'environnement immédiat de l'ADN, et pour l'étape chimique, tous les taux de réaction des espèces radicalaires entre elles et avec l'ADN et les protéines composant les histones.

\subsection{Transport Monte Carlo au niveau de l'ADN}

Les figures 5 et 6 sont assez explicites. Le trajet d'une particule dans ce milieu est une succession de traversées de milieux homogènes (eau environnante) ou hétérogènes (structures de molécules biologiques). Cette méthode pourra se généraliser à la fibre chromosomique qui est un ensemble d'ADN linéaire, de protéines et de nucléosomes prenant une structure identique à celle des plasmides (voir également Fig. 12).

\subsection{Modélisation des réactions chimiques primaires}

À la fin de l'étape physique et jusqu'à environ $10^{-8}$ à $10^{-6}$ seconde après le passage du rayonnement, les radicaux issus de la radiolyse du milieu environnant diffusent et peuvent réagir entre eux et avec les constituants de l'ADN. Ceci est réalisé dans le code CPA100 par une modélisation contrôlée par la diffusion et les équations de Smoluchowski : les espèces diffusent et réagissent lorsqu'elles se trouvent à une distance inférieure au rayon de réaction évalué au moyen de taux de réaction expérimentaux. Ceci permet de prendre réellement en compte l'environnement de l'ADN et de s'adapter aux conditions expérimentales pour valider le code ou tester 
M. TERRISSOL

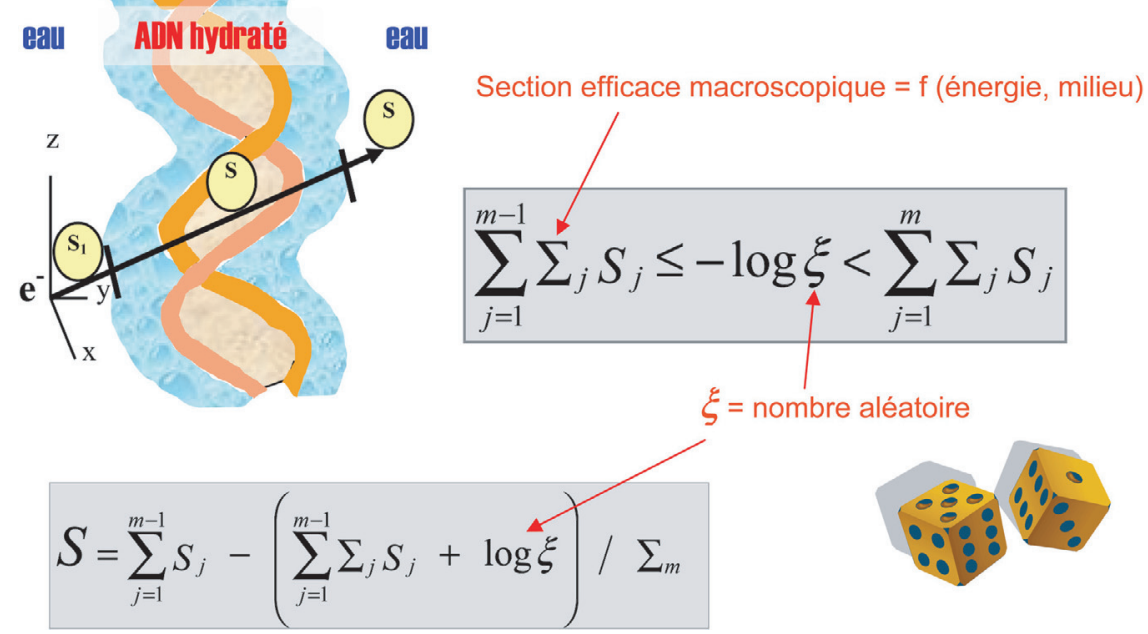

Figure 5 - Échantillonnage des trajets dans le milieu hétérogène. Path sampling in heterogeneous medium.
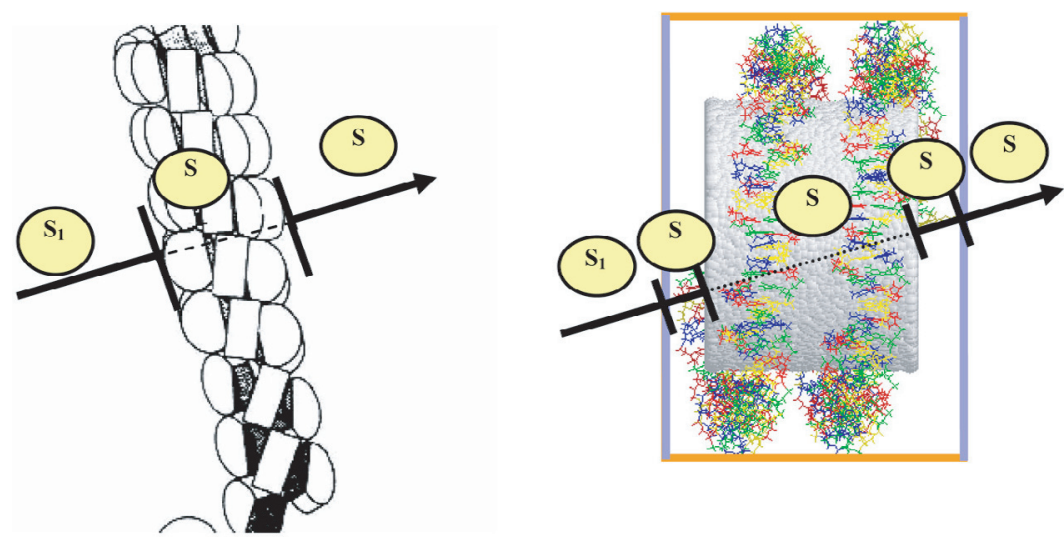

1- un nucléosome est sélectionné ;

2- un nucléotide et 8 des ses voisins sont sélectionnés ;

3- un atome ou une molécule est sélectionné(e) parmi les 9 nucléotides.

Figure 6 - Sélection d'un nucléosome, puis d'un nucléotide.

Nucleosome (core particle) selection, then nucleotide selection. 


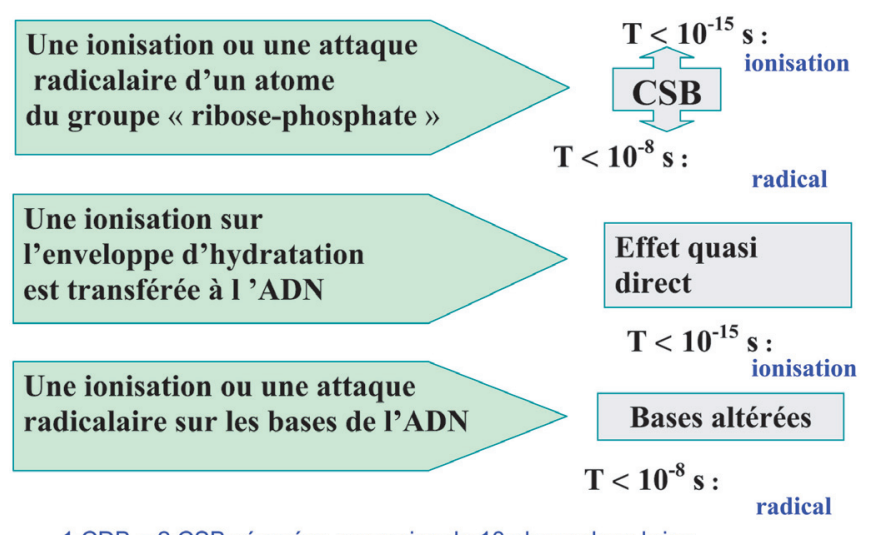

$1 \mathrm{CDB}=2 \mathrm{CSB}$ séparées par moins de $10 \mathrm{pb}$ sur deux brins

Figure 7 - Relations interactions-dommages utilisées dans ce modèle.

Damage pathways used in this model.

des hypothèses. Plus de 150 réactions sont modélisées et les radicaux et espèces prises en comptes sont :

- $\mathrm{OH}^{\bullet}, \mathrm{H}_{3} \mathrm{O}^{+}, \mathrm{e}^{-}$aq $, \mathrm{H}^{\bullet}, \mathrm{H}_{2}, \mathrm{HO}_{2}{ }^{\bullet}, \mathrm{ONOO}-, \mathrm{HO}_{2}^{-}, \mathrm{NO}^{\bullet}, \mathrm{H}_{2} \mathrm{O}_{2}$, etc. ;

- inhibiteurs de radicaux (DMSO, TRIS, etc.);

- sous unités de l'ADN (bases, sucres-phosphate);

- protéines histones.

\section{4. Évaluation des dommages}

Les principaux dommages de l'ADN sont les cassures simple brin (CSB) et l'altération des bases. On voit sur la figure 7 comment sont pris en compte ces dommages. Leur distribution dans l'espace et dans le temps, fonction de la séquence génétique étudiée, sera proposée aux radiobiologistes. Une Cassure Double Brin consiste en deux CSB sur brins opposés.

\section{Exemples d'applications}

\subsection{Cartographie des ionisations par rayons $X$ ultra-mous}

L'irradiation d'un brin d'ADN linéaire dans un milieu aqueux par des rayons $\mathrm{X}$ de très faible énergie permet d'étudier la sensibilité de celui-ci et de comparer effets directs et indirects (Fig. 8). 


\section{Cartographie des photoionisations}

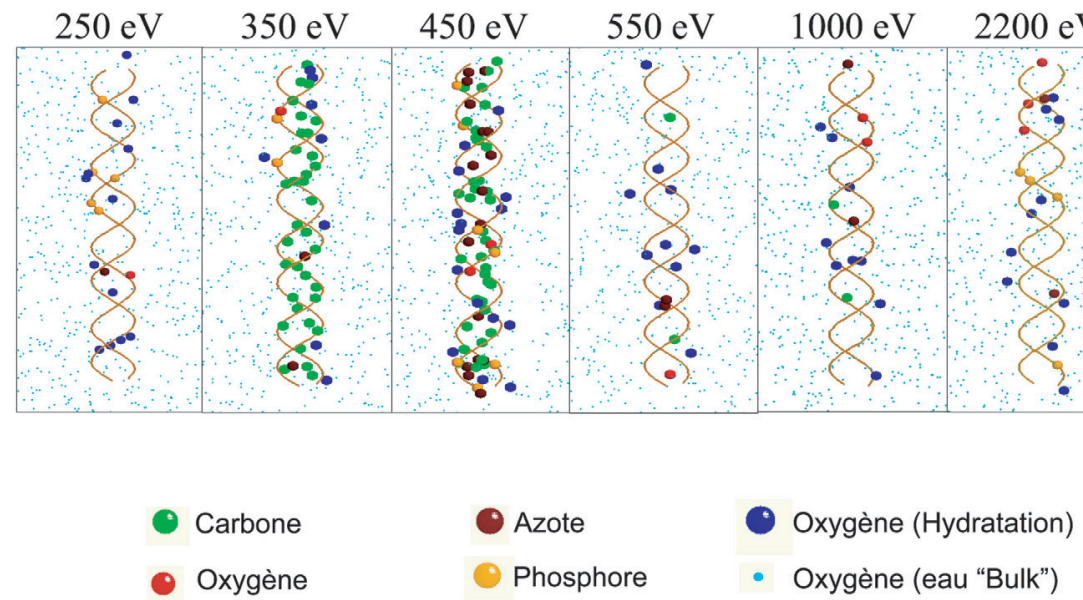

Figure 8 - Ionisations directes créées par des rayons $X$ ultra mous de diverses énergies.

Direct ionisations created by ultrasoft $X$-rays of various energies.

\subsection{Photoactivation du cisplatine}

Le cisplatine est un anti-cancéreux qui va se fixer sur l'ADN des cellules malignes et détruit cet $\mathrm{ADN}$ malade de telle manière qu'il ne puisse pas continuer à proliférer. Or le rendement n'est pas égal à un et le cisplatine est un produit toxique à effets secondaires. La présence d'un atome lourd (le Z du platine vaut 79) a permis d'imaginer sa photoactivation. En irradiant avec des photons légèrement supérieurs au niveau $\mathrm{K}$ du platine, la vacance produite entraîne une réorganisation avec émission d'une cascade d'électrons Auger qui vont à leur tour détruire l'ADN et « finir le travail ». Tout ceci permettant de rendre les traitements plus efficaces, de diminuer les doses toxiques et ainsi d'améliorer le confort du malade. On peut voir sur la figure 9 la position que prend le cisplatine dans l'ADN. On irradie ensuite avec des photons d'environ $80 \mathrm{keV}$.

Sur la figure 10 , on a les contributions des divers niveaux du platine à l'émission d'électrons Auger après vacance K. L'encart indique à quoi contribueront les divers groupes d'électrons ou de rayons $\mathrm{X}$ créés. Les électrons les plus dommageables pour l'ADN seront les électrons d'énergie inférieure à 


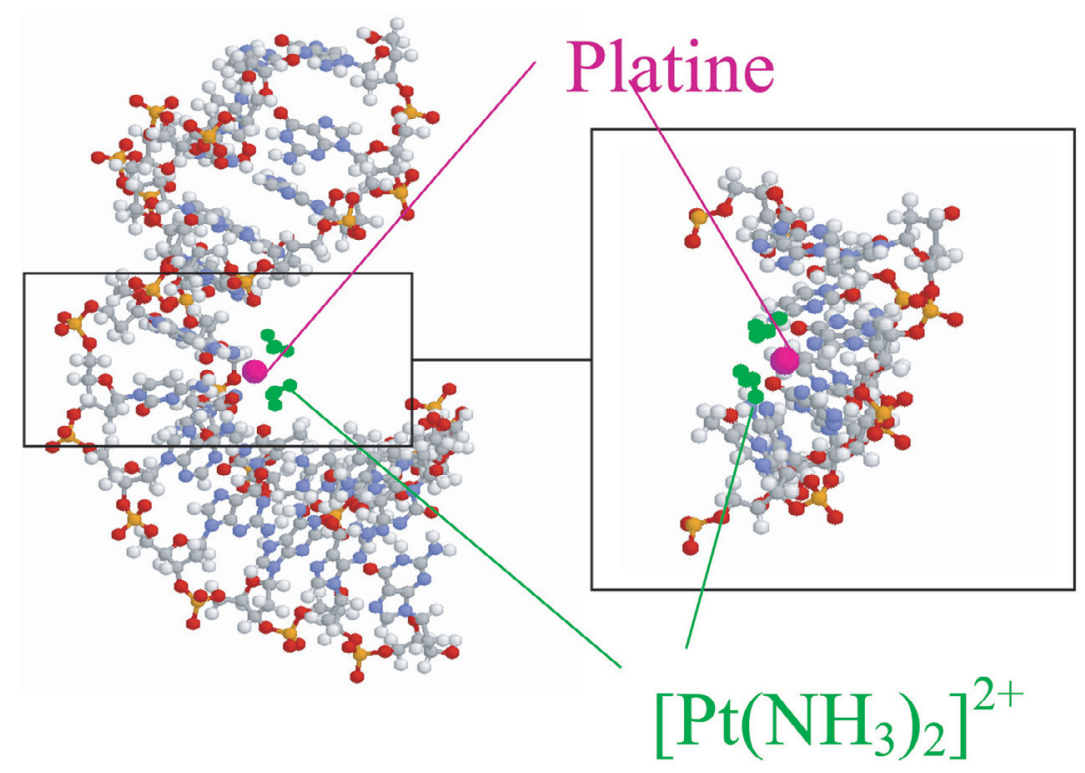

Figure 9-Position du cisplatine dans l'ADN.

Cisplatine position in DNA.

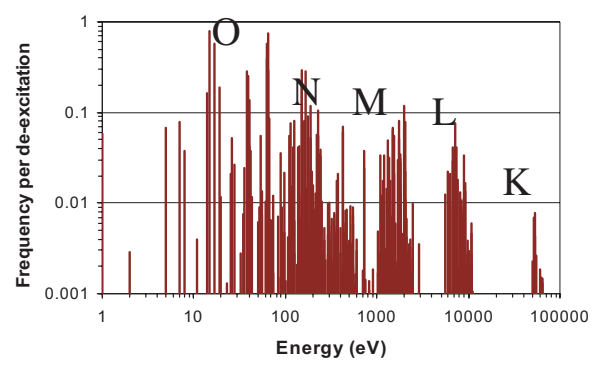

électrons :

0-400 eV : effet direct.

Autour de $700 \mathrm{eV}$ : effet indirect.

3-13 keV : fibre voisine.

$60 \mathrm{keV}$ : ionisation \& excitation

au long du parcours.

rayons $X$ :

$68 \mathrm{keV}$ : émission sans

interactions locales.

Nombre moyen d'électrons Auger : 9,77

Nombre moyen de photons émis : 1,51

Figure 10 - Spectre d'émission du platine après vacance sur la couche $\mathrm{K}$.

Emission spectrum after platinum K-vacancy. 
Sans Cis-Pt

\begin{tabular}{|l|r|}
\hline Rien & 0,9414 \\
\hline CSB & 0,0443 \\
\hline CSB + & 0,0076 \\
\hline 2 CSB & 0,0021 \\
\hline CDB & 0,0036 \\
\hline CDB + & 0,0008 \\
\hline CDB ++ & 0,0002 \\
\hline
\end{tabular}

Avec Cis-Pt

\begin{tabular}{|l|c|}
\hline Rien & 0,9458 \\
\hline CSB & 0,0409 \\
\hline CSB+ & 0,0059 \\
\hline 2 CSB & 0,0017 \\
\hline CDB & 0,0043 \\
\hline CDB+ & 0,0011 \\
\hline CDB++ & 0,0003 \\
\hline
\end{tabular}

\section{CDB sont $29 \%$ plus complexes avec le Cis-Pt}

Figure 11 - Complexité des dommages induits par la vacance d'un électron $\mathrm{K}$ du platine.

Damage complexity induced by platinum K-vacancy.

$1 \mathrm{keV}$, les plus faibles participant davantage à l'attaque directe des brins, alors que ceux d'environ $700 \mathrm{eV}$ créeront des radicaux qui réagiront par la suite avec l'ADN. Les électrons d'énergie plus grande et les rayons $\mathrm{X}$ émis interagiront plus loin ou bien sortiront de l'organisme, participant avec une très faible probabilité à des dommages supplémentaires.

Les tableaux de la figure 11 montrent le degré de complexité des dommages : ce sont les fréquences observées pour une vacance :

- rien indique aucun effet observé ;

- CSB indique une seule cassure sur un seul brin d'ADN ;

- $\mathrm{CSB}+$ indique une CSB plus une base altérée ;

- 2 CSB indique deux cassures sur le même brin ;

- $\quad \mathrm{CDB}$ indique une CSB sur chaque brin ;

- $\mathrm{CDB}+$ indique une CDB plus une base altérée ou une CSB ;

- $\mathrm{CDB}++$ indique tous les autres cas à fort dommage.

L'ensemble de ces résultats permet de dire que l'apport du cisplatine photoactivé augmente l'efficacité de $29 \%$.

\subsection{Perspectives}

La figure 12 permet d'évaluer le chemin à accomplir : le but étant de modéliser la fibre chromosomique, les chromosomes et au-delà la cellule, afin de déterminer les distributions de tous les dommages primaires. 


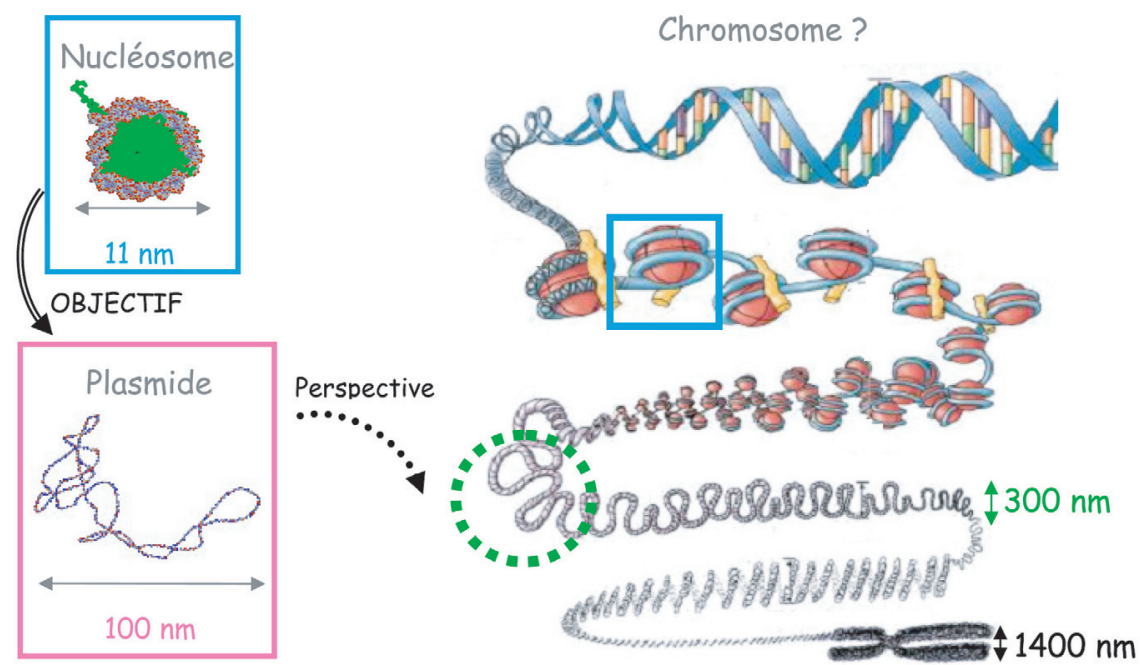

Figure 12 - Extension de la modélisation aux chromosomes.

Extension of the model to chromosomes.

\section{Principales publications au cours des 30 dernières années}

Nous ne pouvons citer ici toutes les publications effectuées dans le cadre de ces recherches depuis une trentaine d'années. L'ensemble des chercheurs ayant contribué au développement de ces travaux sont Mesdemoiselles et Messieurs : A. Beaudre, V. Caudrelier, M.A. Combes, M. Demonchy, S. Edel, T. Eudaldo, J. Fourmenty, J.P. Mannens, C. Martin, A. Peudon, M. Roch, A. Saifi, M. Tep et J.M. Vrigneaud.

Nikjoo H., O'Neill P., Goodhead D.T., Terrissol M. (1997) Computational Modelling of low-energy electron-induced DNA damage by early physical and chemical events, Int. J. Radiat. Biol. 71, 467-483.

Peudon A., Edel S., Terrissol M. (2006) Molecular basic data calculation for electron and photon transport in chromatin, Radiat. Prot. Dosim., doi: 10.1093/rpd/ncl452.

Pomplun E., Terrissol M. (1994) Low-energy electrons inside active DNA models: a tool to elucidate the radiation action mechanisms, Rad. Env. Biophys. 33, 279-292.

Pomplun E., Demonchy M., Terrissol M. (1997) Auger Electron Action Inside Hydrated DNA and Nucleosome Models, in Microdosimetry, An Interdisciplinary Approach. The Royal Society of Chemistry, Cambridge, pp. 19-22.

Terrissol M. (1978) Méthode de simulation du transport d'électrons d'énergies comprises entre 10 eV et $30 \mathrm{keV}$, Thèse de l'université de Toulouse III.

Terrissol M. (1994) Modelling of radiation damage by I-125 on a nucleosome, Int. J. Radiat. Biol. 66, 447-451.

Terrissol M., Patau J.P. (1980) Simulation complète des trajectoires d'électrons de quelques dizaines de keV dans les métaux, J. Microsc. Spectr. Elec. 5, 383-391. 
M. TERRISSOL

Terrissol M., Beaudre A., Caudrelier V. (1987) Simulation of spatial and temporal evolution of chemical species created by electrons and photons in liquid water. In: 8th International Congress of Radiation Research, Edimbourg. Taylor \& Francis, p. 48

Terrissol M., Beaudre A. (1990) Simulation of space and time evolution of radiolytics species induced by electrons in water, Radiat. Prot. Dosim. 31, 175-177.

Terrissol M., Pomplun E. (1994) Computer simulation of DNA incorporated 125 Iodine Auger cascades and of the associated radiation chemistry in aqueous solution, Radiat. Prot. Dosim. 52, 177-181.

Terrissol M., Vrigneaud J.M. (2001) Analogue Monte Carlo to model radiation induced DNA damage. In: Advanced Monte Carlo for Radiation Physics, Particle Transport Simulation and Applications (A. Kling, F. Barao, M. Nagakawa, L.M.N. Távora, P. Vaz, Eds) pp. 261-266, ISBN 3-540-41795-8, Springer Verlag.

Terrissol M., Martin C., Pomplun E. (2002) Computer Simulation of ${ }^{57} \mathrm{Fe}-$ Bleomycin Auger Effects in DNA, Radiat. Prot. Dosim. 99, 69-72.

Terrissol M., Edel S., Pomplun E. (2004) Computer evaluation of direct and indirect damages induced by free and DNA-bounded iodine-125 in the chromatin fibre, Int. J. Radiat. Biol. 80, 905-908. 